\title{
Effect of an obesogenic diet on circadian activity and serum hormones in old monkeys
}

\author{
Henryk F Urbanski ${ }^{1,2,3,4}$, Kevin Mueller ${ }^{2}$ and Cynthia L Bethea ${ }^{1,2,5}$ \\ ${ }^{1}$ Division of Neuroscience, Oregon National Primate Research Center, Beaverton, Oregon, USA \\ ${ }^{2}$ Division of Reproductive \& Developmental Sciences, Oregon National Primate Research Center, \\ Beaverton, Oregon, USA \\ ${ }^{3}$ Department of Behavioral Neuroscience, Oregon Health \& Science University, Portland, Oregon, USA \\ ${ }^{4}$ Department of Physiology \& Pharmacology, Oregon Health \& Science University, Portland, Oregon, USA \\ ${ }^{5}$ Department of Obstetrics \& Gynecology, Oregon Health \& Science University, Portland, Oregon, USA
}

Correspondence

should be addressed

to H F Urbanski

Email

urbanski@ohsu.edu

\begin{abstract}
Like women, old female rhesus macaques undergo menopause and show many of the same age-associated changes, including perturbed activity/rest cycles and altered circulating levels of many hormones. Previous studies showed that administration of an estrogen agonist increased activity in female monkeys, that hormone therapy (HT) increased activity in postmenopausal women and that obesity decreased activity in women. The present study sought to determine if postmenopausal activity and circulating hormone levels also respond to HT when monkeys are fed a high-fat, highsugar Western style diet (WSD). Old female rhesus macaques were ovo-hysterectomized $(\mathrm{OvH})$ to induce surgical menopause and fed a WSD for 2 years. Half of the animals received estradiol-17 $\beta(\mathrm{E})$, beginning immediately after $\mathrm{OvH}$, while the other half received placebo. Animals in both groups showed an increase in body weight and a decrease in overall activity levels. These changes were associated with a rise in both daytime and nocturnal serum leptin concentrations, but there was no change in serum concentrations of either cortisol or dehydroepiandrosterone sulfate (DHEAS). These data suggest that 2 years of HT has little or no effect on locomotor activity or circadian hormone patterns in menopausal macaques fed an obesogenic diet.
\end{abstract}

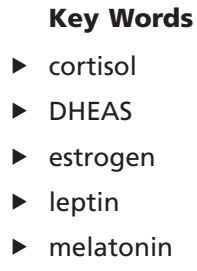

Endocrine Connections (2017) 6, 380-383

\section{Introduction}

Like elderly women, old female rhesus macaques show a marked decline in circulating E concentrations (1), and several monkey studies have demonstrated the therapeutic potential of $\mathrm{E}$ or a selective estrogen receptor modulator (SERM) supplementation on age-associated cognitive decline and activity $(2,3,4,5,6)$. However, the monkeys in the above studies were fed a standard primate chow that was low in fat and sugar, but high in micronutrients. Therefore, it is unclear if age-associated perturbations of activity-rest cycles and alterations in circadian hormone patterns are exacerbated after menopause by maintenance on a typical high-fat, high-sugar WSD and, more importantly, if these changes can be overcome by E supplementation. In the present study, we examined these issues using a nonhuman primate model of surgical menopause, in which old rhesus macaques were $\mathrm{OvH}$ and fed a WSD for 2 years. Although expected changes in body weight, level of activity and circulating hormone levels were observed, there was no obvious difference between the E-treated animals and placebo controls. http://www.endocrineconnections.org
DOI: $10.1530 /$ EC-17-0108 (c) 2017 The authors Published by Bioscientifica Ltd

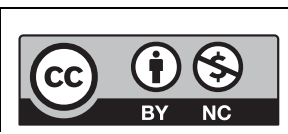

This work is licensed under a Creative Commons Attribution-NonCommercial 4.0 International License. 


\section{Materials and methods}

\section{Animals}

This study was approved by the Oregon National Primate Research Center (ONPRC) Institutional Animal Care and Use Committee and used 14 old (>20 years) female rhesus macaques (Macaca mulatta). The animals were housed indoors in pens (3-4 animals per pen) under controlled environmental conditions: $24^{\circ} \mathrm{C}$ temperature; 12-h light and 12-h darkness photoperiods (lights on at 07:00h) and were cared for by the ONPRC Division of Comparative Medicine in accordance with the National Research Council's Guide for the Care and Use of Laboratory Animals. Daily meals at 08:00 h and 15:00 h were supplemented with fresh fruits or vegetables; fresh drinking water was available ad libitum. At Month 0 , the animals' diet (Monkey Diet, LabDiet, Inc., St Louis, MO, USA) was replaced with unlimited access to a typical American diet or WSD (TAD Primate Diet; LabDiet, Inc.), which provided calories with 36\% fat, 44\% carbohydrates (includes $18.5 \%$ sugars) and $18 \%$ protein. In comparison, regular monkey chow provides calories with 13\% fat, $69 \%$ complex carbohydrates (includes 6\% sugars) and 18\% protein. Additional enrichment included watching video programs and interactions with the Behavioral Science Unit staff and animal care technicians.

\section{Experimental design}

Approximately 6 weeks after the start of the WSD, all of the animals were OvH. Half $(n=7)$ were immediately started on HT in the form of E-containing silastic capsules, which achieved serum E concentrations of $82.1 \pm 0.9 \mathrm{pg} /$ $\mathrm{mL}$; the other half $(n=7)$ received empty capsules (placebo), which achieved serum E concentrations of $6.6 \pm 0.2 \mathrm{pg} / \mathrm{mL}$ on average over 2 years. Serum E was measured every 2 months and the capsule replaced or its size adjusted as deemed appropriate. Animals that showed abnormally low activity prior to WSD were excluded in order to determine whether there was an effect of $\mathrm{E}$ on activity. Blood samples were collected from each animal under ketamine $\mathrm{HCl}$ sedation $(10 \mathrm{mg} / \mathrm{kg}$ body weight, intramuscular), at Month -1 and again at Month 24; the serum was stored frozen at $-20^{\circ} \mathrm{C}$ and subsequently assayed for leptin, cortisol and DHEAS. Note, because many hormones show a circadian pattern of secretion (7) two blood samples were collected at each time point, one at $\sim 08: 00 \mathrm{~h}$ and one at $20: 00 \mathrm{~h}$.

\section{Monitoring of 24-h activity}

As previously described (8), each animal was fitted with an Actiwatch activity monitor (Philips-Respironics), worn inside a protective case that was attached to a lightweight loose-fitting aluminum collar (Primate Products, Inc., Immokalee, FL, USA). At the start of the study, 24-h activity recordings were made for 10 consecutive days and a characteristic baseline pattern was established for each animal; activity was again monitored at the end of the study. The actograms were subsequently analyzed using Actiware-Sleep (version 3.4) software (Cambridge Neurotechnology Ltd, Cambridge, UK). The mean total daily activity (defined as the average 24-h activity) was calculated for each animal, as was the mean daytime activity (defined as activity during the period between 07:00 $\mathrm{h}$ and 19:00 h) and mean nighttime activity (activity between 19:00 h and 07:00h).

\section{Hormone measurements}

Plasma leptin concentrations were determined by RIA (Linco Research) with a detection limit of $0.25 \mathrm{ng} / \mathrm{mL}$. The intra-assay coefficients of variation were $5.6 \%$ at $3.5 \mathrm{ng} / \mathrm{mL}$ and $6.0 \%$ at $21.1 \mathrm{ng} / \mathrm{mL}$. The corresponding inter-assay coefficients of variation were 7.9 and $7.0 \%$ respectively. E was assayed using a Roche Diagnostics Cobas e411 automatic clinical platform assay instrument. The intra-assay and inter-assay coefficients of variation were all less than $10 \%$ and the assay detection limit was $5 \mathrm{pg} /$ $\mathrm{mL}$. Cortisol was assayed by electro-chemiluminescence using the Elecsys 2010 Platform (Roche Diagnostics). DHEAS was assayed using RIA with a highly specific antibody for DHEAS-17-(O-carboxymethyl)oxime-BSA (Endocrine Services, Tarzana, CA, USA) and [3H]DHEAS (SA, 22 Ci/mmol). For the cortisol and DHEAS assays, the intra-assay and inter-assay coefficients of variation were less than $10 \%$ and the assay detection limits were $3 \mathrm{ng} / \mathrm{mL}$.

\section{Statistical analysis}

RM ANOVAs followed by Fisher's LSD procedure were used to assess the effects of time and to compare mean values from the same animals at Month -1 and Month 24 . $P<0.05$ was considered to be significant. http://www.endocrineconnections.org
DOI: 10.1530/EC-17-0108
(C) 2017 The authors Published by Bioscientifica Ltd
This work is licensed under a Creative Commons Attribution-NonCommercial 4.0 International License. 
Table 1 Effects of Western style diet in aged female rhesus macaques.

\begin{tabular}{|c|c|c|c|c|c|c|}
\hline & \multicolumn{2}{|c|}{ Pooled data } & \multicolumn{2}{|c|}{ OvH + placebo } & \multicolumn{2}{|c|}{ OvH + E } \\
\hline & Month -1 & Month 24 & Month -1 & Month 24 & Month -1 & Month 24 \\
\hline Body weight (kg) & $7.3 \pm 0.3$ & $9.04 \pm 0.4 * * *$ & $7.5 \pm 0.5$ & $9.3 \pm 0.4^{\# \#}$ & $7.1 \pm 0.4$ & $8.8 \pm 0.7^{\# \#}$ \\
\hline 24-h activity & $139 \pm 10.9$ & $115 \pm 11.3 * *$ & $118 \pm 7.5$ & $93.4 \pm 7.7^{\#}$ & $160 \pm 17.7$ & $136 \pm 18.5^{\#}$ \\
\hline Daytime activity & $245 \pm 20.1$ & $201 \pm 20.0 * *$ & $207 \pm 13.7$ & $165 \pm 16.0^{\#}$ & $283 \pm 34.6$ & $237 \pm 32.3^{\#}$ \\
\hline Nighttime activity & $33 \pm 3.1$ & $28 \pm 3.4$ & $30 \pm 4.7$ & $22 \pm 1.3$ & $36 \pm 4.1$ & $34 \pm 6.0$ \\
\hline \multicolumn{7}{|l|}{ Hormones } \\
\hline Leptin (ng/mL) AM & $5.3 \pm 0.6$ & $13.3 \pm 1.3 * * *$ & $6.2 \pm 0.7$ & $15.5 \pm 2.0^{\# \#}$ & $4.3 \pm 1.0$ & $11.1 \pm 1.4^{\# \#}$ \\
\hline Leptin (ng/mL) PM & $6.7 \pm 1.0$ & $14.0 \pm 1.2 * * *$ & $7.1 \pm 1.4$ & $16.4 \pm 1.9^{\# \#}$ & $6.2 \pm 1.5$ & $11.6 \pm 1.0^{\#}$ \\
\hline Cortisol (ng/mL) AM & $250 \pm 28.5$ & $252 \pm 17.9$ & $276 \pm 40$ & $254 \pm 25$ & $219 \pm 40$ & $250 \pm 28$ \\
\hline Cortisol (ng/mL) PM & $128 \pm 17.5$ & $153 \pm 17.6$ & $145 \pm 23$ & $137 \pm 25$ & $111 \pm 27$ & $169 \pm 25$ \\
\hline DHEAS (ng/mL) AM & $92.9 \pm 14.7$ & $76.4 \pm 13.9$ & $75.7 \pm 17.7$ & $65.9 \pm 15.5$ & $113.0 \pm 23.2$ & $87.0 \pm 23.7$ \\
\hline DHEAS (ng/mL) PM & $47.9 \pm 7.6$ & $58.6 \pm 12.3$ & $41.0 \pm 11.8$ & $43.7 \pm 9.5$ & $54.9 \pm 9.6$ & $73.4 \pm 22.1$ \\
\hline
\end{tabular}

Values represent means \pm S.E.M. of 7 animals.

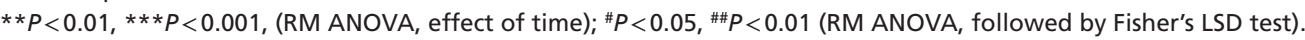

$\mathrm{E}$, estradiol-17 $\beta ; \mathrm{OvH}$, ovo-hysterectomized.

\section{Results}

Differences in body weight, activity patterns and serum hormone concentrations, between Month -1 and Month 24 are depicted in Table 1; the table also depicts pooled data from the two time points. Body weight showed a significant $(P<0.001)$ increase after 2 years of maintenance on a WSD. In addition, overall activity as well as daytime activity $(P<0.01)$, but not nocturnal activity, showed a significant decrease in all animals (Fig. 1). There was a $17 \%$ decrease in combined groups, a $20 \%$ decrease in the $\mathrm{OvH}+$ placebo group and a $15 \%$ decrease in the $\mathrm{OvH}+\mathrm{E}$ group in overall activity. For daytime activity only, there was an $18 \%$ decrease in combined groups, a $20 \%$ decrease in the $\mathrm{OvH}+$ placebo group and a $16 \%$ decrease in the $\mathrm{OvH}+\mathrm{E}$ group. There appeared to be little difference in the reduction of activity between the two groups. The associated circadian hormones showed expected
$\mathrm{AM} / \mathrm{PM}$ differences, with serum leptin levels being highest at night, and serum cortisol and DHEAS levels being highest during the day. However, there was no significant change between Month -1 and Month 24 for the two adrenal steroids (cortisol and DHEAS), whereas leptin showed a significant $(P<0.001)$ increase during both the day and night. Overall, there were no significant differences in activity or hormone responses shown by the placebo- and E-treated monkeys fed WSD for 2 years.

\section{Discussion}

Two-thirds of the US population is considered to be overweight or obese, and this may adversely affect the responses of postmenopausal women to HT. In female rhesus macaques fed standard primate chow, SERM treatment caused weight loss with an increase in physical
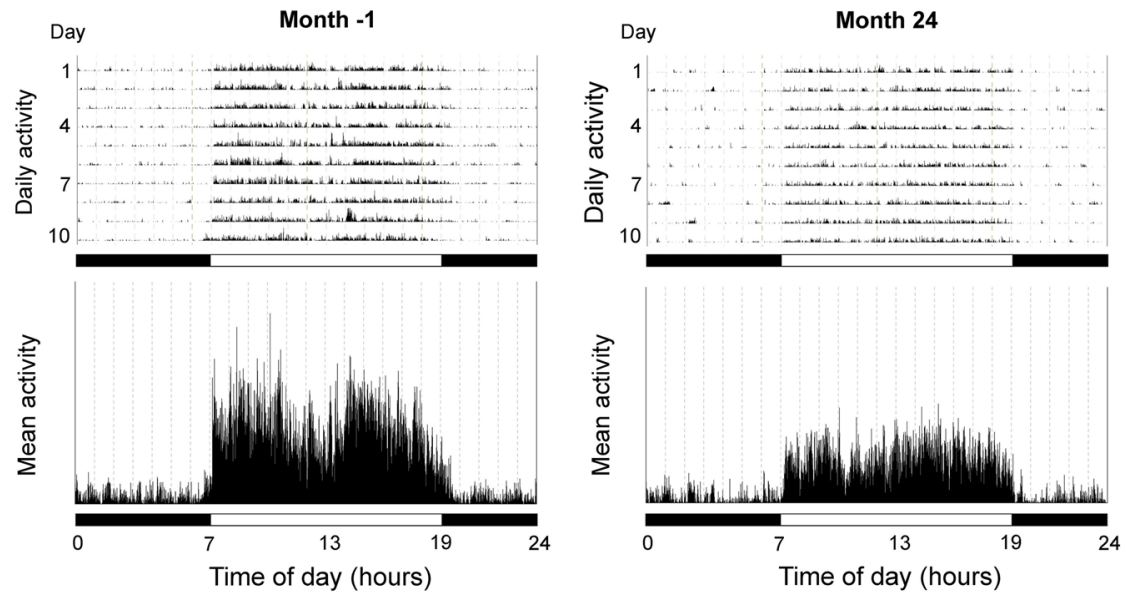

Figure 1

Representative actograms from an old female rhesus macaque maintained on a WSD. Activity recordings were made at Month -1

(i.e., 1 month before exposure to a WSD and 2.5 months before $\mathrm{OvH}$ ), and again at Month 24 (i.e., 25 months later). In the upper panels, the height of the vertical lines is indicative of the intensity of physical activity at any particular time of day; the mean 24-h activity profiles across the 10 consecutive days are depicted in the lower panels. The horizontal black and white bars correspond to the times of night and day, respectively.

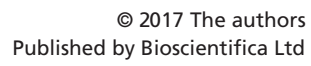

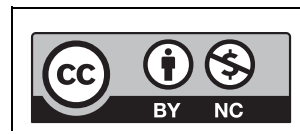

This work is licensed under a Creative Commons Attribution-NonCommercial 4.0 International License. 
activity (5). Similarly, postmenopausal women using HT exhibited higher activity than non-users $(9,10)$, and of note, physical activity was reduced in postmenopausal women with $\mathrm{BMI} \geq 30$ compared to women with $\mathrm{BMI}<30$ (11). In the present study, HT appeared to have no effect on final body weight or serum leptin levels, and little effect on activity levels in WSD-fed monkeys. The mechanism by which WSD and/or obesity block the effect of HT needs further investigation, but systemic cytokines are markedly elevated in obesity (12) and they may play a role. In addition, the E-treated animals and the placebo controls showed little difference in the responses of their circadian serum hormone levels to a WSD. In conclusion, WSD and the accompanying obesity prevented HT from elevating overall activity relative to controls. Serum leptin increased with body weight and HT had no effect on either parameter. However, WSD did not impact the circadian nature of 3 hormones examined. These data suggest that HT may be ineffective in obese postmenopausal women depending on the outcome examined.

\section{Declaration of interest}

The authors declare that there is no conflict of interest that could be perceived as prejudicing the impartiality of the research reported.

\section{Funding}

This work was supported by National Institutes of Health grants AG-029612, AG-036670, OD-011092 and OD-011895.

\section{Author contribution statement}

H F U and C L B designed the study, analyzed the data and prepared the manuscript. $\mathrm{K} \mathrm{M}$ executed the study.

\section{Acknowledgements}

The authors are grateful to the ONPRC Division of Comparative Medicine for assistance with care of the animals and to the ONPRC Endocrine Technology Support Core for performing the hormone assays.

\section{References}

1 Downs JL \& Urbanski HF. Neuroendocrine changes in the aging reproductive axis of female rhesus macaques (Macaca mulatta). Biology of Reproduction 200675 539-546. (doi:10.1095/ biolreprod.106.051839)

2 Rapp PR, Morrison JH \& Roberts JA. Cyclic estrogen replacement improves cognitive function in aged ovariectomized rhesus monkeys. Journal of Neuroscience 20032 5708-5714.

3 Lacreuse A. Effects of ovarian hormones on cognitive function in nonhuman primates. Neuroscience 2006138 859-867. (doi:10.1016/j. neuroscience.2005.09.006)

4 Voytko ML, Higgs CJ \& Murray R. Differential effects on visual and spatial recognition memory of a novel hormone therapy regimen of estrogen alone or combined with progesterone in older surgically menopausal monkeys. Neuroscience 2008154 1205-1217. (doi:10.1016/j.neuroscience.2008.04.064)

5 Sullivan EL, Shearin J, Koegler FH \& Cameron JL. Selective estrogen receptor modulator promotes weight loss in ovariectomized female rhesus monkeys (Macaca mulatta) by decreasing food intake and increasing activity. American Journal of Physiology: Endocrinology and Metabolism 2012302 E759-E767. (doi:10.1152/ajpendo.00327.2011)

6 Kohama SG, Renner L, Landauer N, Weiss AR, Urbanski HF, Park B, Voytko ML \& Neuringer M. Effect of ovarian hormone therapy on cognition in the aged female rhesus macaque. Journal of Neuroscience 201636 10416-10424. (doi:10.1523/JNEUROSCI.0909-16.2016)

7 Urbanski HF \& Sorwell K. Age-related changes in neuroendocrine rhythmic function in the rhesus macaque. Age 201234 1111-1121. (doi:10.1007/s11357-011-9352-z)

8 Urbanski HF. Circadian variation in the physiology and behavior of humans and nonhuman primates. In Animal Models of Behavioral Analysis, Neuromethods 50, Chapter 9, pp 217-235. Ed J Raber. New York, NY, USA: Humana Press, 2011.

9 Bowen RS, Turner MJ \& Lightfoot JT. Sex hormone effects on physical activity levels: why doesn't Jane run as much as Dick? Sports Medicine 201141 73-86. (doi:10.2165/11536860-000000000-00000)

10 Weinberg N, Young A, Hunter CJ, Agrawal N, Mao S \& Budoff MJ. Physical activity, hormone replacement therapy, and the presence of coronary calcium in midlife women. Women and Health 201252 423-436. (doi:10.1080/03630242.2012.682705)

11 Menzel J, di Giuseppe R, Wientzek A, Kroke A, Boeing H \& Wekert C. Physical activity, bone health, and obesity in peri-/pre- and postmenopausal women: results from the EPIC-Potsdam study. Calcified Tissue International 201597 376-384. (doi:10.1007/s00223015-0027-0)

12 Debnath M, Agrawal S, Agrawal A \& Dubey GP. Metaflammatory responses during obesity: pathomechanism and treatment. Obesity Research and Clinical Practice 201610 103-113. (doi:10.1016/j. orcp.2015.10.012)

Received in final form 8 June 2017

Accepted 15 June 2017

Accepted Preprint published online 15 June 2017 http://www.endocrineconnections.org

DOI: 10.1530/EC-17-0108
() 2017 The authors Published by Bioscientifica Ltd

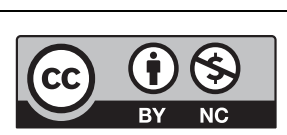

This work is licensed under a Creative Commons Attribution-NonCommercial 4.0 International License. 